ARTICLE

DOI: $10.1038 / s 41467-018-03642-w$

\title{
Velocity correlated crack front and surface marks in single crystalline silicon
}

\author{
Lv Zhao (1) ${ }^{1}$, Didier Bardel ${ }^{1}$, Anne Maynadier ${ }^{1,2} \&$ Daniel Nelias (i] ${ }^{1}$
}

Single crystalline silicon fractures on low-energy cleavage planes such as (111) and (110). The crack propagation cannot accurately be predicted by linear elastic fracture mechanics since it does not account for small scale and inelastic phenomena such as atomic lattice trapping. Here we show that, under pure bending load, (110) cleavage in silicon single crystal rapidly accelerates to $3700 \mathrm{~m} / \mathrm{s}$ without crack path deviation or crack branching, contrasting previous observations. We highlight that the crack front shape involves strong velocity dependence and presents a curvature jump during very high-speed crack growth. In addition, we observe special marks-a kind of periodic surface undulation-that exclusively arise on the rapid fracture surfaces, and we suggest that they are front wave traces resulting from an intrinsic local velocity fluctuation. This finding gives insight to the wavy nature of the crack front in the absence of material asperity.

\footnotetext{
${ }^{1}$ Univ Lyon, INSA-Lyon, CNRS UMR5259, LaMCoS, Lyon F-69621, France. ${ }^{2}$ Present address: Department of Applied Mechanics, FEMTO-ST Institute, Univ Bourgogne Franche Comté, CNRS/UFC/ENSMM/UTBM, Besançon F-25000, France. Correspondence and requests for materials should be addressed to L.Z. (email: Iv.zhao08@gmail.com) or to D.N. (email: daniel.nelias@insa-lyon.fr)
} 
T he fracture behavior of silicon has received substantial attention over the last few years in relation to its anisotropic behavior, and also for a very local mechanism called atomic lattice trapping which influences the early stage of crack propagation $^{1,2}$. The fracture of silicon is also of high interest for solar cell applications, given that the solar cells are subjected to vibrations and impacts during transportation and mounting prior to using, and also to severe thermal cycles, hail impact forces and wind vibration during operation.

Single crystalline silicon breaks up preferentially on low-energy crystallographic planes such as (111) and $(110)^{3-7}$. The brittle characteristic makes the fracture a rapid dynamic process with a propagation velocity comparable to the surface wave speed ${ }^{8-11}$. Slow crack growth $(\ll 2000 \mathrm{~m} / \mathrm{s})$ has barely been observed in experiments ${ }^{6,8}$, indicating the existence of a threshold crack velocity in silicon. The velocity gap between 0 and the threshold is attributed to a localized phase transformation before reaching the critical load ${ }^{12}$. This local atom rearrangement actively blunts the crack tip and necessitates a higher energy flux than the intrinsic material toughness upon the crack initiation. The fracture along the [100] direction on the (110) plane (shortened as (110) [100]) cannot be achieved; a systematical deflection onto a (111) plane has been observed in tensile tests ${ }^{13}$. Pérez and Gumbsch ${ }^{14}$ provided an explanation for this deflection phenomenon by means of quantum mechanical calculations. The atomistic fracture scenario revealed that the bond breaking process along (110) [100] path is discontinuous and can cause a large lattice trapping ${ }^{15}$. Another kind of large plane deflection-from (110) [110] to (111) - has been observed under 3 -line bending condition ${ }^{11,16}$. This deflection involved velocity dependence and occurred when the crack propagated faster than $1700 \mathrm{~m} / \mathrm{s}$. The authors explained the deflection as a result of the dynamic toughness evolution, based on a postulated phonon emission mechanism ${ }^{17}, 18$. However, according to the aforementioned mechanism, the dynamic toughness of the (110) crystal plane is markedly different from the experimentally measured one ${ }^{8}$. This difference acts against the generalization of the postulated theory and thus the explanation for the plane-switch scenario. Yet, the discussion on the deflection origin has never incorporated the contact effect, note that the 3-line bending introduces a complex stress distribution in the vicinity of the contact line-which is also the region where the crack path deviation has been observed.

Whereas the global fracture behavior of silicon has been widely investigated, the local fracture behavior is less known and needs further exploration, especially under bending load where the crack front is curved and a local crack velocity gradient is present. Fracture surface marks constitute the only way to reconstruct a high-speed fracture process since they are highly correlated with the crack front ${ }^{19-22}$. The primary surface marks are Wallner lines ${ }^{23,24}$, which involve the interaction between shear waves and the propagating crack front. Wallner lines emanate essentially from surface defects of the specimen or external ultrasonic sources ${ }^{25}$. Secondary fracture surface marks can be attributed to front wave (FW) traces. FWs are solitary waves that propagate only along the crack front at approximately the Rayleigh wave speed $\left(C_{\mathrm{R}}\right)$. Initially, these waves have been numerically predicted and depicted as propagating velocity fluctuations confined to the fracture plane 26,27 . The first experimental observation of FW traces has been reported by Sharon et al. ${ }^{28,29}$ when exploring the fracture surface of brittle soda-lime glass plates in tensile tests. However, this dissertation led to severe polemics in the fracture community. Bonamy and Ravi-Chandar ${ }^{24}$ suggested that the highlighted marks in refs. ${ }^{28,29}$ could completely be interpreted as Wallner lines. Indeed, when a solid fractures under tensile loading, FW traces and Wallner lines would have very similar shapes because of the straight crack front and the close velocities of the two source waves ${ }^{28}$. In the experiments of Sharon and co-workers, due to the material asperity introduced at middle thickness of the sample, FWs and shear waves would emanate simultaneously from the same spot and then overlap one another. The generated surface marks therefore cannot be isolated for meticulous identification, albeit their decay characteristics are differen $\mathrm{t}^{30}$. Hence, exploration and discussion on FW observation remain open.

This work focuses on both global and local fracture behaviors of (110) [110] cleavage within a silicon single crystal, under pure bending. Overall crack path and fracture surface marks are addressed in combination with crack steady-state velocity. We show that (110) [110] cleavage neither involves cleavage plane deflection nor crack branching, even in an extremely high-speed fracture process $(3700 \mathrm{~m} / \mathrm{s})$. This finding is contradictory with the aforementioned observations in 3-line bending tests. Thanks to meticulous fractographic analysis, it is for the first time highlighted that the crack front shape is strongly dependent on the crack velocity and involves a curvature jump when propagating faster than $2800 \mathrm{~m} / \mathrm{s}$. Jointly with the curvature jump, special surface marks (SSMs) have been observed on the fracture surface. These marks are periodic undulations and involve significantly different features from the Wallner lines (also present), and we propose that they are FW traces resulting from a fracture toughness induced local velocity fluctuation.

\section{Results}

Stability of (110) [110] cleavage. Thanks to various pre-crack sizes (from about 200 to $1000 \mu \mathrm{m}$ ) and a substantial amount of experimental data (90 tests), a large range of crack steady-state velocities have been obtained, from about $1050 \mathrm{~m} / \mathrm{s}\left(0.23 C_{R}\right)$ to $3700 \mathrm{~m} / \mathrm{s}\left(0.83 C_{\mathrm{R}}\right)$, as shown in Fig. 1a. The steady-state velocities are obtained thanks to high-speed imaging technique as follows: the crack-tip position is highlighted by performing subtractions between sequential images after cracking and the last image before cracking. The subtraction results are then subjected to contrast reinforcement as well as denoising processing (Matlab Wavelet algorithm) to have an accurate crack length determination, as shown in Fig. 1b, $\mathrm{c}$ for a representative slow crack and a rapid crack, respectively. The crack velocity is calculated by analyzing the results image after image, based on a time step of $5.6 \mu \mathrm{s}$. The velocity uncertainty is about $100 \mathrm{~m} / \mathrm{s}$ while the uncertainty on the crack-tip location is five pixels (equivalent to $0.6 \mathrm{~mm}$ ). Here the representative slow crack corresponds to a velocity of $1200 \mathrm{~m} / \mathrm{s}$ whereas the rapid one propagates at $3600 \mathrm{~m} / \mathrm{s}$. The two cracks involve well-distinguished fracture surface morphologies, as can be noticed in Fig. 1d, e. Results for other cracks are summarized in Supplementary Fig. 1. Both slow and rapid cracks propagate on the (110) plane without noticeable deflection onto the (111) plane (see Fig. 2a for the rapid crack). In fact, no deflection has been observed on any primary crack path (initiated from the pre-crack) in our wide set of experimental data presented in Fig. 1a. The deflection free (110) [110] cleavage up to $3700 \mathrm{~m} / \mathrm{s}$ in this work contradicts what has been reported previously; however, only observed under 3-line bending condition $^{11,16}$. One may assume that the deflection revealed in previous works is linked to the complex stress gradient generated by the contact with the punch roller. Conversely, we believe our observations are more reliable, since the cracks undergo a contact-free stress field for 4-line bending. Indeed, the stress field below the contact line may promote lateral shearing, which impacts the crack path. This assumption has been verified in our work: when the primary crack propagated at around $3600 \mathrm{~m} / \mathrm{s}$, secondary cracks nucleated underneath the punch rollers. These cracks tended to propagate on a (111) plane, as shown in Fig. 2b. Note that the measured tilt angle is about $33^{\circ}$, close to the 
a

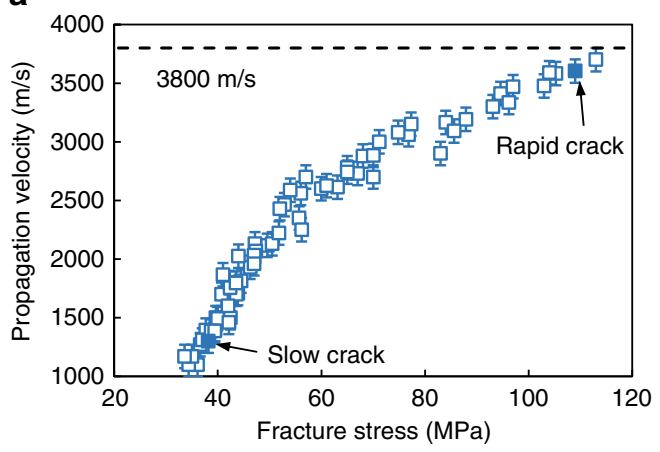

b

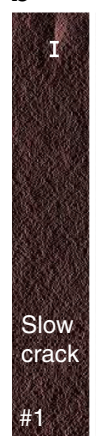



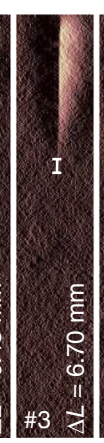

$\mathbf{C}$

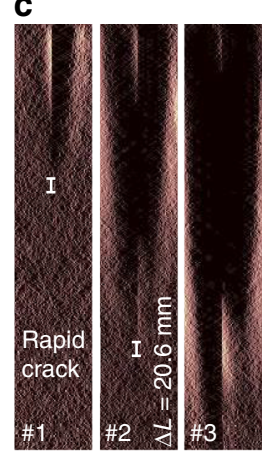

e
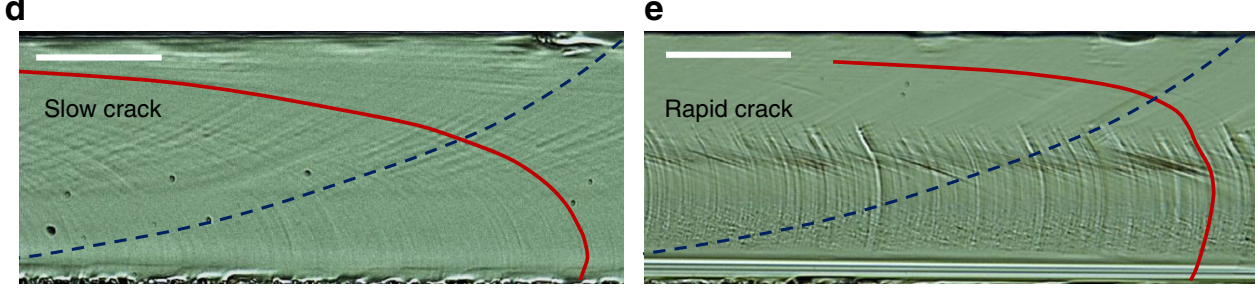

Fig. 1 (110) cleavage of silicon single crystal with different crack propagation velocities. a Synthesis of the crack speed measurements. b, c Successive image subtractions from the one prior to cracking, for a slow and a rapid crack, identified by the two solid squares in $\mathbf{a}$. The white bar reveals the uncertainty for the crack-tip position (five pixels). d, e Relatively smooth crack surface for the crack that propagated at a slow speed and the rough one for the fast propagation, respectively. The red solid lines and blue dashed ones highlight the Wallner lines and atomic debonding paths, respectively. The cracks propagate from the left to the right. Scale bars: $100 \mu \mathrm{m}$

a

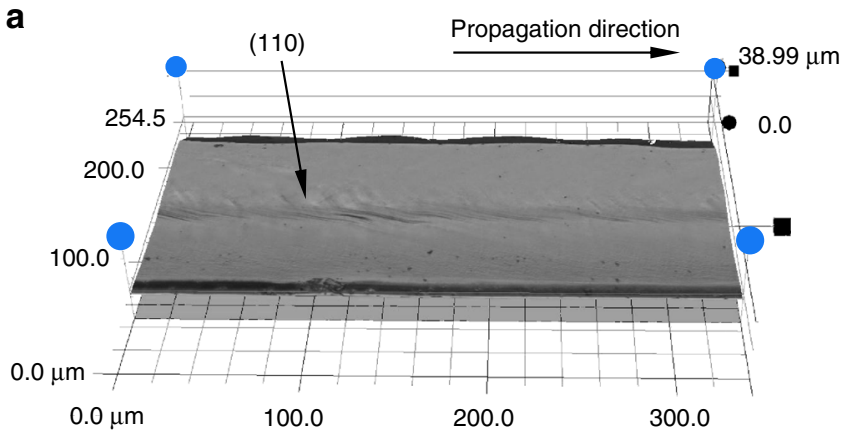

b

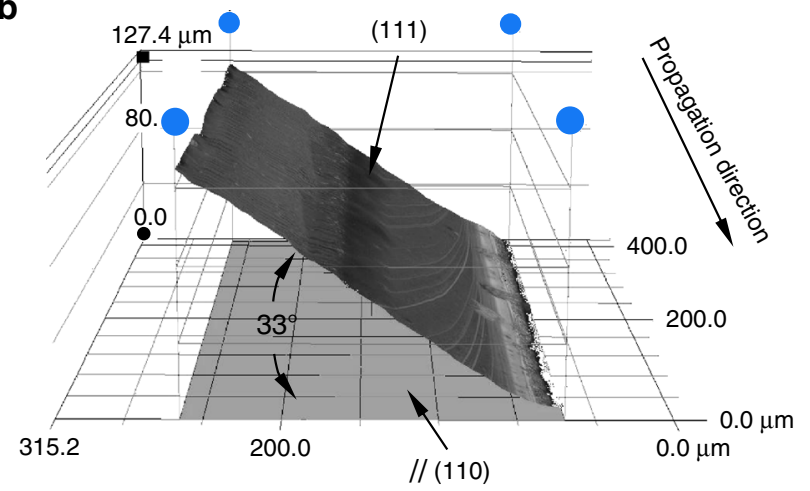

Fig. 2 Crack surface topography for the fast crack that propagated at 3600 $\mathrm{m} / \mathrm{s}$. a Primary crack that initiates from the pre-crack. b Secondary crack after deviating underneath the punch roller

theoretical angle between the (110) and (111) planes, i.e., $35.2^{\circ}$. Therefore, we can conclude that the (110) [110] cracking is very stable in perturbation-free regions, and that the deflection phenomenon is a result of contact effect rather than thermal phonon emission. On the other hand, the proposed phonon emission mechanism ${ }^{17,18}$ should be considered with caution.

Crack front evolution. In our bending configuration, the crack front is curved due to the stress gradient in the specimen thickness, and the local velocity will decrease from the tensile side to the compression side. In such a case, the knowledge of the crack front shape can help us to assess the local velocities and investigate the local fracture behavior which can only be inferred by postmortem analysis with fracture surface marks. In the smooth region, both (slow crack and rapid crack in Fig. 1b, c) fracture surfaces contain successive-hook like marks initiated from lower surface defects, as shown in Fig. 1d, e. These marks are very typical Wallner lines in bending fracture tests ${ }^{16,31}$. By observing the Wallner line shape and knowing the Rayleigh wave velocity ${ }^{32}$ as well as the crack propagation speed $v$, the real crack front shape can be addressed in the inverse sense of Wallner line generation. The method is based on the principle such that each point on the Wallner line belongs to the crack front during its propagation. As schematized in Fig. 3a, we choose a fixed time increment $\Delta t$ and discretize the Wallner line from the lower part to the upper part with a couple of $(n)$ surface waves, then we conduct horizontal translations from the last discretized point to the lowest point, with translation distances $0, \Delta t \cdot v, 2 \Delta t \cdot v, \ldots$, $n \Delta t \cdot v$. This way we will get $n$ new points, including the last discretized point on the Wallner line. The crack front shape can be finally obtained by connecting these new points. With this method, the crack fronts for the slow crack $(1200 \mathrm{~m} / \mathrm{s})$ and the rapid one $(3600 \mathrm{~m} / \mathrm{s})$ as well as for two additional cracks that propagated at intermediate velocities have been determined and plotted in Fig. 3b. The front for the slowest crack can be approximated with an ellipse of semi-axes $a=3 h$ and $b=0.85 h$ ( $h$ stands for the specimen thickness), which agrees well with the conclusion inferred from a propagation velocity lower than 1700 $\mathrm{m} / \mathrm{s}$ by Sherman and Be'ery ${ }^{16}$. This previous work did not address the front shape for any higher crack velocity since the fracture 
experiments involved a deflection from the (110) plane to the (111) plane, where the surface instabilities ${ }^{33}$ concealed the Wallner lines. Nevertheless, thanks to the stable propagation in a contact-free region, crack front shapes for rapid propagation cases $(>2000 \mathrm{~m} / \mathrm{s})$ are for the first time highlighted in this work. From Fig. 3b, one can notice that the front shape of high-speed crack is complex and cannot be described by a simple basic shape or function, mainly due to the curvature jump at a certain height. A meticulous analysis has permitted to identify the critical speed at which a curvature jump can be observed. This curvature jump onset velocity is about $2700-2800 \mathrm{~m} / \mathrm{s}$. Another intriguing observation involves the significant evolution of the crack front shape as a function of the crack propagation velocity under identical loading conditions. This velocity dependence feature is related to our bending configuration and not expected in tensile one. Note that the crack front is directly linked to the atomic debonding process, i.e., local velocity; the front shape evolves as

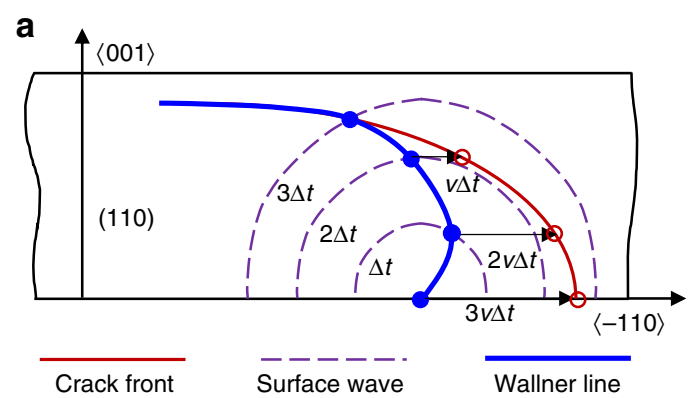

b

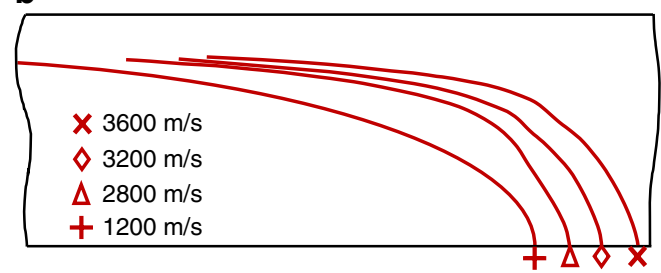

Fig. 3 Crack front identification, method and results. a Method for recovering the crack front shape from Wallner lines and surface waves. The blue solid line denotes the crack front, the dashed ones stand for the propagating surface waves, and the red one represents the Wallner line. $\mathbf{b}$ Crack front shapes for a slow crack propagating at $1200 \mathrm{~m} / \mathrm{s}$ and a rapid one at $3600 \mathrm{~m} / \mathrm{s}$, along with cracks propagating at intermediate velocities. Note that the front for rapid cracks presents a curvature jump while that for the slowest one is smooth long as the local velocity distribution alters. Under bending condition, the local crack velocity at the tensile side (or lowest point along the crack front) coincides with the global crack velocity, which is also the one measured by real-time diagnostics. Then it decreases and tends toward 0 in the upper region since the crack front tends to an asymptotic horizontal line (see Fig. 3b). For instance, for any two cases with different global velocities, the local velocity distributions are markedly different across the specimen thickness. Consequently, the crack front changes from one case to the other as it accommodates a dissimilar velocity distribution. Thanks to the determined crack front shape and the measured global crack velocity, it is then possible to address the local crack velocity as well as the energy release rate at any locus of the crack front. In practice, the consideration of velocity correlated crack front shape is of tremendous importance in order to ensure high accuracy in such analysis. Moreover, the crack front shape in (111) cleavage should be determined in a dedicated fracture analysis rather than referring that in (110) cleavage at the same global crack velocity, given that the material toughness is quite different between the two crystal planes ${ }^{5}$. However, these two points have not been properly taken into account in the previous studies ${ }^{16,33}$.

Secondary special surface marks. Particularly, for the rapid crack $(3600 \mathrm{~m} / \mathrm{s})$, besides the Wallner lines, one may observe some SSMs and eventually some local tiny instabilities. These marks arise just after the crack initiation and cover continuously the whole crack surface. In fact, SSMs have been systematically observed on the fracture surface of specimens when the crack propagation velocity is higher than $2800 \mathrm{~m} / \mathrm{s}$, jointly with the presence of a curvature jump along the crack front shape. According to the observations of fracture surfaces in the steadystate phase (see Supplementary Fig. 1), these special marks arise at about $0.34 h$ with a tilt angle of $30^{\circ}$ for $2800 \mathrm{~m} / \mathrm{s}$, while at about $0.58 \mathrm{~h}$ with a tilt angle of $12^{\circ}$ for $3700 \mathrm{~m} / \mathrm{s}$. Both the onset height and the tilt angle involve a strong dependence on the crack velocity, as summarized in Supplementary Fig. 2. This dependence can be more clearly noticed on the fractography near the crack initiation point for the rapid crack $(3600 \mathrm{~m} / \mathrm{s})$. As presented in Fig. 4, the SSMs appear $150 \mu \mathrm{m}$ away from the crack initiation site, their vertical position then moves up from about $0.35 h$ to $0.55 h($ at $1000 \mu \mathrm{m})$ as the crack propagation velocity increases up to the steady-state regime with a stable position of $0.57 \mathrm{~h}$.

In order to address more accurately the geometrical features of the SSMs, we focus our attention on the sample with a crack velocity of $3600 \mathrm{~m} / \mathrm{s}$. A representative region is illustrated in Fig. 5a with a digital microscopic image, and then detailed with

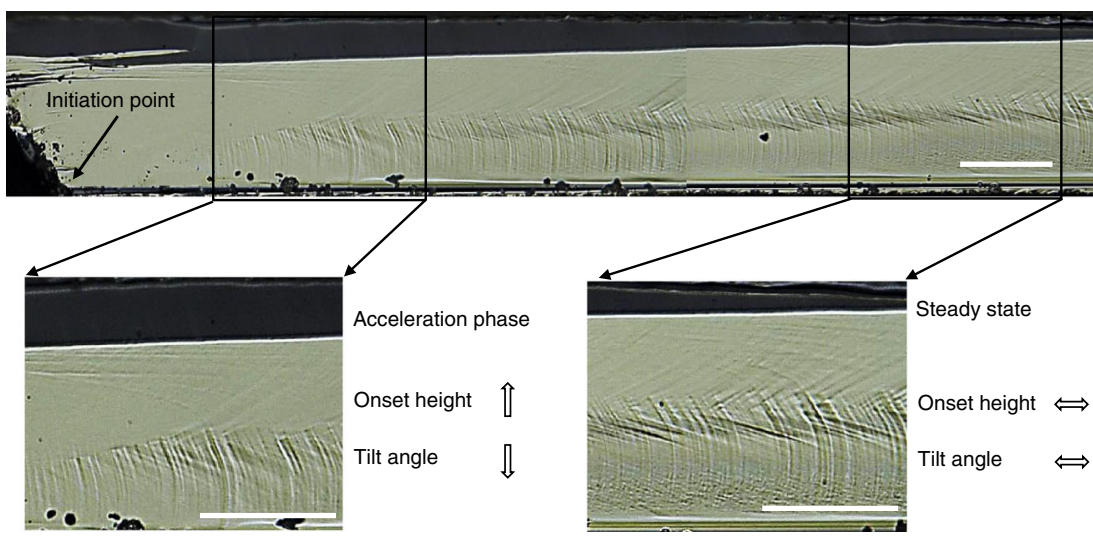

Fig. 4 Crack surface morphology nearby the crack initiation spot for the rapid crack ( $3600 \mathrm{~m} / \mathrm{s})$. SSMs appear about $150 \mu \mathrm{m}$ away from the initiation site and their position moves up in thickness as the crack velocity increases. Scale bars: $100 \mu \mathrm{m}$ 
a
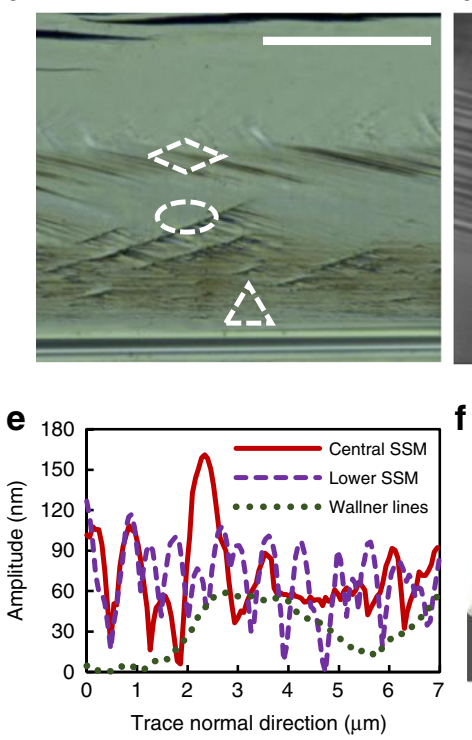

b

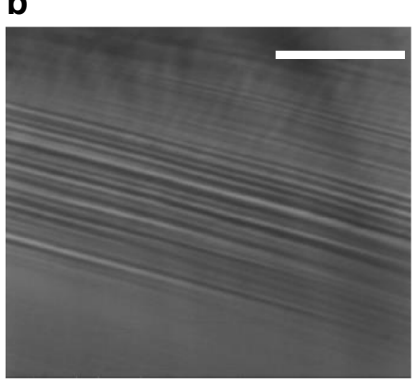

f

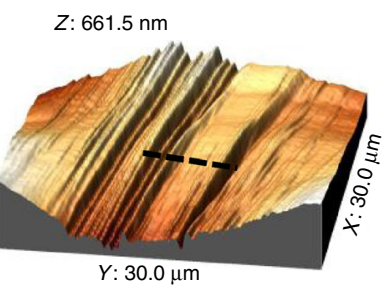

c

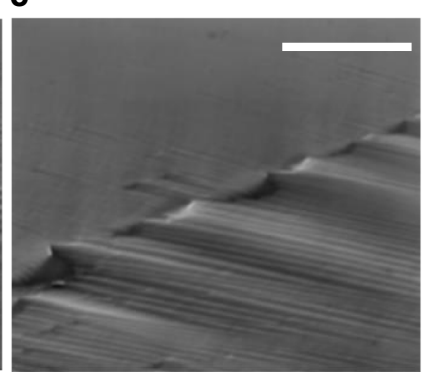

g

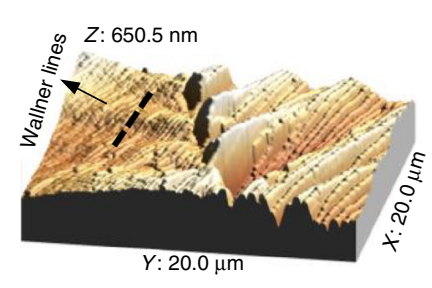

d

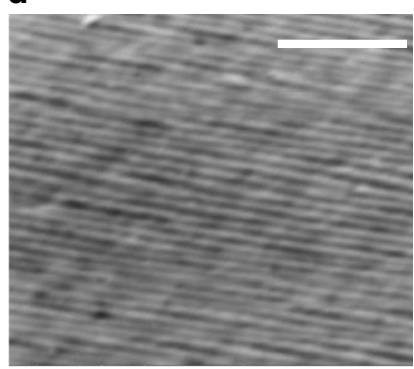

$\mathbf{h}$

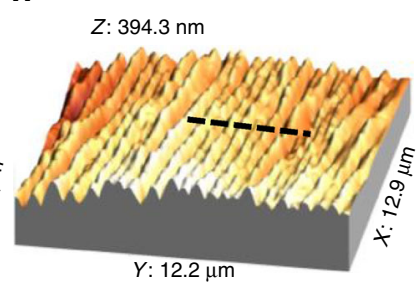

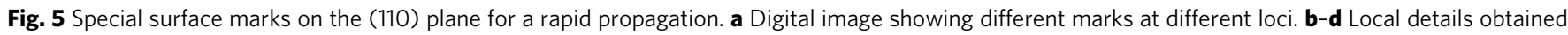
by SEM which correspond to the framed regions in parallelogram, ellipse, and triangle, respectively. e Profile (wavelength and amplitude) of the Wallner lines and the SSMs measured along the dashed lines presented in $\mathbf{f}-\mathbf{h}$. $\mathbf{f}-\mathbf{h}$ Topographies of the SSM in the regions corresponding to $\mathbf{b}-\mathbf{d}$. Scale bars: $\mathbf{a}$ (100 $\mu \mathrm{m}), \mathbf{b}(10 \mu \mathrm{m}), \mathbf{c}(10 \mu \mathrm{m})$, and $\mathbf{d}(5 \mu \mathrm{m})$

SEM observations in Fig. 5b-d and AFM topographies in Fig. $5 f-h$ (the SEM images and AFM topographies have up-down correspondence in Fig. 5). According to Fig. 5b, the central SSMs are almost straight and tilted by $12^{\circ}$ from the horizontal direction. This angle allows us to rule out the interpretation of SSM based on dislocation. The lower SSMs all present a similar tilt angle, as shown in Fig. 5d. They seem to be extension of the central SSMs. Tiny instabilities eventually arise as presented in Fig. 5c. AFM topographies provide more readable characteristics of the SSMs at the same regions. Figure $5 \mathrm{f}, \mathrm{h}$ shows that the SSMs are in fact small-amplitude surface undulations rather than micro-plane deflections. Concerning the local instabilities or marked undulations that one may observe in Fig. $5 \mathrm{~g}$, it can be noticed that they appear at the intersections between the atomic debonding path and the Wallner lines. These local perturbations from the Wallner lines (underlying shear waves) generate locally more important SSMs whose amplitude increases strongly and then decays rapidly until they sink in lower SSMs. With the profile plots (Fig. 5e) carried out along the black dashed lines presented in Fig. 5f-h, we can notice that the central SSMs are similar to the lower ones in terms of spacing $(0.5$ vs. $0.5 \mu \mathrm{m})$ and amplitude (100 vs. $100 \mathrm{~nm})$. Spacing between Wallner lines is larger, about $5 \mu \mathrm{m}$ (see also Fig. 1d, e).

What can be the source of these SSMs that involve a strong velocity dependence? In our experiments, the silicon is of high purity (99.9999\%), and no inclusions or voids have been observed on the fracture surfaces, so the SSMs are not a consequence of crack front-material defect interaction. They are not likely to initiate from the Rayleigh waves either, given the well distinguished spatial frequencies between the SSMs and the Wallner lines (see Fig. 5e). Moreover, the SSMs cannot be interpreted as multiple Wallner lines ${ }^{25}$, since the latter are usually observed when the crack initiates from a defect inside the solid, or in a case where external ultrasonic sources are present, both irrelevant to our fracture experiments. Another key argument to eliminate the material defects and Rayleigh waves is that they utterly fail to explain the velocity dependence of the SSM onset as well as the onset height in thickness. At this stage, we propose that the SSMs are intrinsic fracture products related to high crack velocity in (110) [110] cleavage. However, this kind of secondary surface marks have never been observed in tensile fracture tests, even for a crack velocity higher than $3000 \mathrm{~m} / \mathrm{s}^{8}$. Hence, the SSM nucleation is likely related to the local crack velocity distribution under bending load.

Front wave mechanism. The local crack velocity is correlated with the crack front shape. Therefore, the curvature jump, which appears jointly with the SSMs (see Fig. 3b), is certainly the manifestation of a local velocity irregularity. Considering the periodic wavy nature of the SSMs as well as their correlation with the presumed velocity irregularity, FW assumption is proposed. Note that FWs are physically localized pulses of in-plane velocity fluctuations, as numerically predicted in ref. ${ }^{26}$. In order to highlight the expected irregularity, the local velocity along the crack front is addressed thanks to the following equation, according to Fig. 6a:

$$
v_{1}=\frac{\sin (\alpha)}{\sin (\alpha+\beta)} \cdot v
$$

Since the curvature jump impacts $\alpha$, it will also modify $v_{1}$. Six cases are now considered for the local velocity plotting, with the global steady-state velocities all higher than $2800 \mathrm{~m} / \mathrm{s}$. The results are shown in Fig. $6 \mathrm{~b}$. The first promising observation is that each considered local velocity profile involves a velocity fluctuation. This evidence constitutes a perfect rationale for the FW assumption in terms of the underlying driving force. More interestingly, it is found that all the fluctuations occur almost at the same local velocity, which is about $2500 \mathrm{~m} / \mathrm{s}$ (see Fig. 6b). This allows us to explain why the SSM onset height rises when the global crack velocity increases, as can be noticed in Fig. $6 \mathrm{~b}$ as well as Supplementary Fig. 2a. It is important to note that the shear waves that generate Wallner lines in a fracture process mainly have two sources, one regards the material flaws which induce an antiplanar shear component, and the other involves an ultrasonic transducer ${ }^{34}$. The in-plane velocity fluctuations therefore would not give rise to Wallner lines, given that mode I loading is 
a

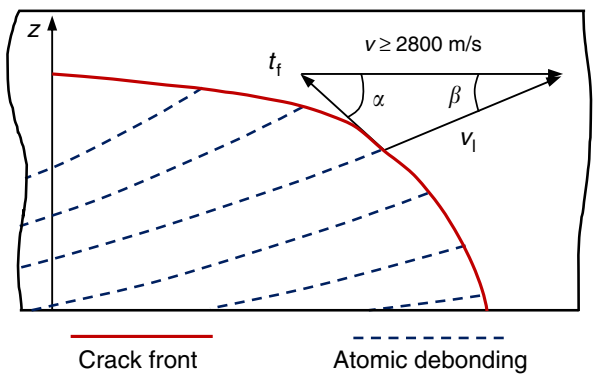

C

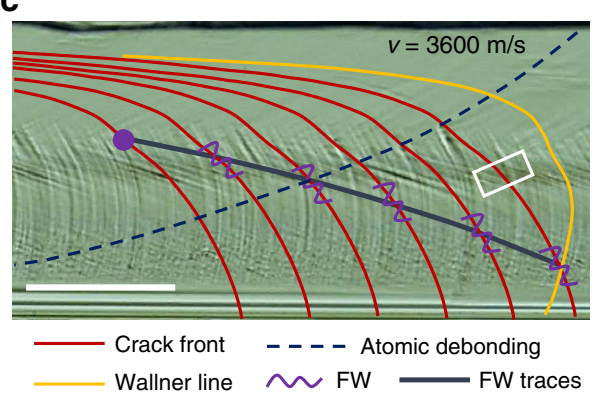

b

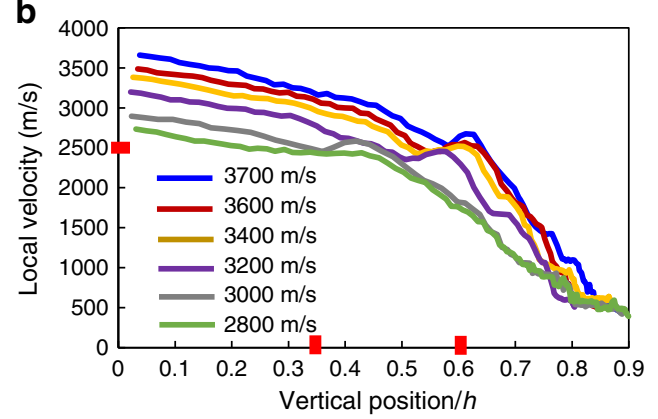

d

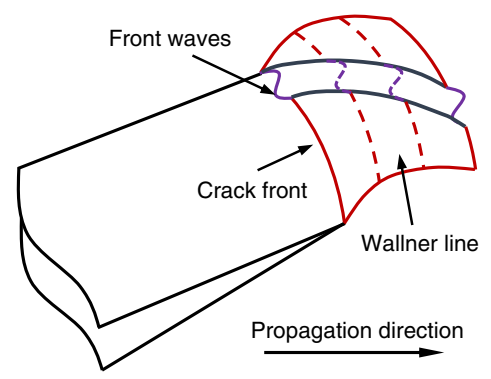

Fig. 6 Analysis of the front wave origin and generation process. a Relationship between the local velocity $v_{l}$ and the macroscopic propagation velocity $v_{\text {, }} t_{\mathrm{f}}$ is the local tangent of the crack front. $\mathbf{b}$ Local velocity profiles with respect to different propagation velocities. $\mathbf{c}$ Real scale schema on the FW generation and propagation, the trace is compared with the experimentally observed SSM. d Superposition of the FW traces on the surface undulation (Wallner lines) in the framed region in c. Scale bar: $100 \mu \mathrm{m}$

maintained and no shear component will be derived. However, the FWs, though emanating from in-plane perturbations, involve an out-of-plane motion due to their nonlinear character ${ }^{28,35}$, and thus produce surface marks, i.e., the so-called FW traces. The FW traces would be locally accompanied by mode III shear due to the out-of-plane components, but not necessarily by the Wallner lines. If Wallner lines were generated, the surface undulations would then be larger and larger in the lower part of the fracture surface because of the continuously generated Wallner lines. This hypothesis cannot be verified since the SSMs involve quite constant amplitudes, as shown in Fig. 5e, h. In order to further verify the FW assumption, the FW traces are produced and compared to the observed SSMs in the steady-state region of the rapid crack $(3600 \mathrm{~m} / \mathrm{s})$. The production incorporates the real crack front shape, the crack velocity, the FW propagation velocity, i.e., the Rayleigh wave speed $C_{R}$ as well as the corresponding curvature jump onset height, i.e., $0.57 \mathrm{~h}$. The comparison is addressed in Fig. $6 c$, a very good agreement can be observed. Another comparison is carried out for a crack velocity of $2800 \mathrm{~m} / \mathrm{s}$, the produced $\mathrm{FW}$ trace is once again coherent with the observed SSM, see Supplementary Fig. 3. As the FW propagates along the crack front, the FW traces will encounter the Wallner lines so that a superimposition of the two surface marks takes place. A schematic is drawn in Fig. 6d, which correlates well with the observation in Fig. $5 \mathrm{~g}$.

It has been highlighted in Fig. 6b that the local velocity fluctuation consistently emerges when the local velocity reaches about $2500 \mathrm{~m} / \mathrm{s}$. This characteristic allows excluding any extrinsic source for the FW nucleation and implies an intrinsic driving force. Here, we propose that the local velocity fluctuation is generated by the sudden increase in the dynamic toughness at a certain velocity, for instance $2600 \mathrm{~m} / \mathrm{s}$ in the (110) [110] cleavage as reported in ref. ${ }^{8}$. In the real fracture scenario, an accommodation will be established between the crack front shape $(\alpha)$, the local crack velocity $\left(v_{1}\right)$, and the static energy release rate $\left(G_{\mathrm{s}}\right)$. Note that $v_{1}$ forms $\alpha, \alpha$ determines $G_{\mathrm{s}}$ under a given loading, while $G_{\mathrm{s}}$ feeds $v_{\mathrm{l}}$, the three interact in a closed circle as illustrated in Supplementary Fig. 4. In our case, due to the presence of the sudden increase in dynamic toughness, the local crack velocity oscillates, and the crack front simultaneously alters to follow the oscillation of the local velocity. The change in the crack front then affects the $G_{s}$ distribution, which in turn adjusts the local velocity. These interactions proceed and terminate until the local velocity evolution along the crack front; the crack front shape and the $G_{\mathrm{s}}$ distribution converge to a mutual accommodation. Through the interactive process, the sudden increase in dynamic toughness intrinsically results in a velocity fluctuation and a curvature jump along the crack front. This in-plane velocity fluctuation then generates FWs which propagate along the crack front and produce the SSMs on the fracture surface.

\section{Discussion}

The FW nucleation has been interpreted in light of the sudden increase in dynamic toughness in the (110) [110] cleavage at $2600 \mathrm{~m} / \mathrm{s}^{8}$. Nevertheless, at the SSM onset site in our case, the local crack direction does not exactly head for the [110] direction, see Fig. 6a. Herein, the authors suppose that such toughness increase exists in other directions between [110] and [111] on the (110) plane at a velocity around $2500 \mathrm{~m} / \mathrm{s}$.

The FWs represent a mode of dissipation ${ }^{28}$. In the absence of external energy source, these waves can only propagate with the strain energy that flows into the crack front. However, in the fracture process under bending, the energy flux in the upper part is low and can barely drive the crack growth above the FW nucleation site, so little or no extra energy would be available for the FW propagation toward the upper part of the crack front. This explains why the SSMs appear only below the FW nucleation site where the energy flux is much higher.

According to our explanation, the FW would emanate for any crack velocity higher than $2500 \mathrm{~m} / \mathrm{s}$, while the FW traces have been experimentally observed only when the crack velocity exceeds $2800 \mathrm{~m} / \mathrm{s}$. It can be seen in Fig. $6 \mathrm{~b}$ that the fluctuation 
becomes smaller as the steady-state velocity decreases and is barely noticeable at $2800 \mathrm{~m} / \mathrm{s}$. Hence, one may assume that the effect of velocity fluctuation is negligible when the steady-state velocity varies between 2500 and $2800 \mathrm{~m} / \mathrm{s}$. In this circumstance, the FWs barely initiate and propagate, which explain why no noticeable traces are left on the fracture surface.

In summary, it is found that (110) [110] cleavage of single crystalline silicon is highly stable and free of plane deflection under pure bending load, even at an extremely high velocity of $3700 \mathrm{~m} / \mathrm{s}$. The crack front shape has been reconstructed from the Wallner lines and revealed to have a strong dependence on the crack velocity. Due to the toughness increase at $2500 \mathrm{~m} / \mathrm{s}$, a local velocity fluctuation arises in high-speed cracks (above $2800 \mathrm{~m} / \mathrm{s}$ ) and manifests as a curvature jump along the crack front. Consequently, FWs emanate following the velocity fluctuation and generate secondary marks on the fracture surface, even in the absence of material asperity. The authors assume that such SSMs-or FW traces-could be observed in other crystalline materials with similar dynamic toughness evolution as in silicon.

\section{Methods}

Sample preparation. The specimens were obtained from solar grade silicon ingot with diamond wire sawing which results in a thickness around $200 \mu \mathrm{m}$ and a square surface of $50 \times 50 \mathrm{~mm}^{2}$. Chemical etching was carried out to eliminate a small layer of the damaged surface, while the saw marks were intentionally kept in order to generate fracture surface marks. A pre-crack was introduced for each specimen with a Vickers indent, employing controlled forces to ensure different defect sizes.

Fracture tests. The fracture tests were carried out using a 4-line bending set up. 4line bending was selected instead of 3-line bending to have a uniform bending stress between the central rollers, and also to get rid of the contact perturbations in the fracture region. The inner and outer spans are $21 \mathrm{~mm}$ and $40 \mathrm{~mm}$, respectively. The specimens were bent to fracture under a quasi-static loading $\left(10^{-6} / \mathrm{s}\right)$ with a LLOYD-Ametek LFPLUS electro-mechanical machine. All the tests were conducted under the same experimental conditions.

A Phantom V710 high-speed camera was set up in order to record the crack process thanks to a $45^{\circ}$ tilted mirror between the two support rollers (refer to ref. ${ }^{22}$ for more information about the experimental setup). The image resolution was fixed to $512 \times 64$ pixels, in combination with an acquisition frequency equal to $180,000 \mathrm{~Hz}$. The camera recording was controlled by a trigger allowing saving the last $2 \mathrm{~s}$ of the images.

Fracture surface evaluation. After the fracture tests, each crack surface was observed with a digital microscope (Keyence VHX-2000) to analyze the fracture surface morphology. High magnification fractographies were obtained with a scanning electron microscope (Oxford Instruments) to highlight tiny fracture surface marks. The nano-metric $3 \mathrm{D}$ information of these marks was obtained thanks to the atomic force microscope AFM PSIA XE-15, with an in plane resolution of $50 \mathrm{~nm}$ and a $1 \mathrm{~nm}$ resolution in height. The AFM topographies were analyzed with the WSxM software ${ }^{36}$.

Data availability. Data supporting the findings of this study are available from the corresponding authors on reasonable request.

Received: 4 August 2016 Accepted: 2 March 2018

Published online: 03 April 2018

\section{References}

1. Cox, B. N., Gao, H., Gross, D. \& Rittel, D. Modern topics and challenges in dynamic fracture. J. Mech. Phys. Solids 53, 565-596 (2005).

2. Bitzek, E., Kermode, J. R. \& Gumbsch, P. Atomistic aspects of fracture. Int. J. Fract. 191, 13-31 (2015).

3. George, A. \& Michot, G. Dislocation loops at crack tips: nucleation and growth-an experimental study in silicon. Mater. Sci. Eng.: A 164, 118-134 (1993).

4. Li, X. et al Anisotropy in fracture of single crystal silicon film characterized under uniaxial tensile condition. Sensors Actuators A: Phys. 117, 143-150 (2005).
5. Pérez, R. \& Gumbsch, P. An ab initio study of the cleavage anisotropy in silicon. Acta Mater. 48, 4517-4530 (2000).

6. Holland, D. \& Marder, M. Ideal brittle fracture of silicon studied with molecular dynamics. Phys. Rev. Lett. 80, 746-749 (1998).

7. Ohring, M. The Materials Science of Thin Films (Academic Press, New York, 1992).

8. Cramer, T., Wanner, A. \& Gumbsch, P. Energy dissipation and path instabilities in dynamic fracture of silicon single crystals. Phys. Rev. Lett. 85, 788-791 (2000).

9. Cramer, T., Wanner, A. \& Gumbsch, P. Crack velocities during dynamic fracture of glass and single crystalline silicon. Phys. Status Solidi A: Appl. Mater. Sci. 164, R5 (1997).

10. Hauch, J., Holland, D., Marder, M. P. \& Swinney, H. L. Dynamic fracture in single crystal silicon. Phys. Rev. Lett. 82, 3823-3826 (1999).

11. Sherman, D. \& Be'ery, I. Velocity dependent crack deflection in single crystal silicon. Scr. Mater. 49, 551-555 (2003).

12. Buehler, M. J., Tang, H., van Duin, A. C. T. \& Goddard, W. A. Threshold crack speed controls dynamical fracture of silicon single crystals. Phys. Rev. Lett. 99, 165502 (2007).

13. Ebrahimi, F. \& Kalwani, L. Fracture anisotropy in silicon single crystal. Mater Sci. Eng.: A 268, 116-126 (1999).

14. Pérez, R. \& Gumbsch, P. Directional anisotropy in the cleavage fracture of silicon. Phys. Rev. Lett. 84, 5347-5350 (2000).

15. Thomson, R., Hsieh, C. \& Rana, V. Lattice trapping of fracture cracks. J. Appl. Phys. 42, 3154-3160 (1971).

16. Sherman, D. \& Be'ery, I. From crack deflection to lattice vibrations-macro to atomistic examination of dynamic cleavage fracture. J. Mech. Phys. Solids 52, 1743-1761 (2004).

17. Atrash, F. \& Sherman, D. Evaluation of the thermal phonon emission in dynamic fracture of brittle crystals. Phys. Rev. B 84, 224307 (2011).

18. Atrash, F. \& Sherman, D. Dynamic fracture instabilities in brittle crystals generated by thermal phonon emission: experiments and atomistic calculations. J. Mech. Phys. Solids 60, 844-856 (2012).

19. Hull, D. Fractography: Observing, Measuring and Interpreting Fracture Surface Topography (Cambridge University Press, Cambridge, 1999).

20. Quinn, G. A history of the fractography of glasses and ceramics. Ceram. Trans. 230, 1-55 (2012).

21. Bradt, R. . Applying fractography and fracture mechanics to the energy and mass of crack growth for glass in the mirror region. J. Eur. Ceram. Soc. 34, 3255-3262 (2014).

22. Zhao, L., Maynadier, A. \& Nelias, D. Stiffness and fracture analysis of photovoltaic grade silicon plates. Int. J. Solids Struct. 97-98, 355-369 (2016).

23. Wallner, H. Linienstrukturen an Bruchflächen. Z. Phys. 114, 368-378 (1939).

24. Bonamy, D. \& Ravi-Chandar, K. Interaction of shear waves and propagating cracks. Phys. Rev. Lett. 91, 235502 (2003).

25. Field, J. Brittle fracture: its study and application. Contemp. Phys. 12, 1-31 (1971).

26. Ramanathan, S. \& Fisher, D. S. Dynamics and instabilities of planar tensile cracks in heterogeneous media. Phys. Rev. Lett. 79, 877-880 (1997).

27. Morrissey, J. W. \& Rice, J. R. Crack front waves. J. Mech. Phys. Solids 46, 467-487 (1998).

28. Sharon, E., Cohen, G. \& Fineberg, J. Propagating solitary waves along a rapidly moving crack front. Nature 410, 68-71 (2001).

29. Sharon, E., Cohen, G. \& Fineberg, J. Crack front waves and the dynamics of a rapidly moving crack. Phys. Rev. Lett. 88, 085503 (2002).

30. Sharon, E., Cohen, G. \& Fineberg, J. Interaction of shear waves and propagating cracks. Phys. Rev. Lett. 93, 099601 (2004).

31. Fréchette, V. D. Failure Analysis of Brittle Materials: Advances in Ceramics (American Ceramic Society, Westerville, OH, 1990).

32. Coufal, H., Meyer, K., Grygier, R. K., Hess, P. \& Neubrand, A. Precision measurement of the surface acoustic wave velocity on silicon single crystals using optical excitation and detection. J. Acoust. Soc. Am. 95, 1158-1160 (1994).

33. Sherman, D., Markovitz, M. \& Barkai, O. Dynamic instabilities in 111 silicon. J. Mech. Phys. Solids 56, 376-387 (2008).

34. Bonamy, D. \& Ravi-Chandar, K. Dynamic crack response to a localized shear pulse perturbation in brittle amorphous materials: on crack surface roughening. Int. J. Fract. 134, 1-22 (2005).

35. Bouchaud, E., Bouchaud, J. P., Fisher, D. S., Ramanathan, S. \& Rice, J. R. Can crack front waves explain the roughness of cracks?. J. Mech. Phys. Solids 50, 1703-1725 (2002)

36. Horcas, I. et al. WSXM: A software for scanning probe microscopy and a tool for nanotechnology. Rev. Sci. Instrum. 78, 013705 (2007).

\section{Acknowledgements}

The authors acknowledge the French research agency ANR for partial funding through the DURASOL Equipex project. We also thank Y. Gaillard from FEMTO-ST Institute fo 
his help on the AFM measurements and our colleague A. Saulot from INSA-Lyon for his support in handling the digital microscope.

\section{Author contributions}

L.Z. and D.B. performed the fracture tests, the crack propagation velocity measurements, the optical fractographies and the crack front shape determination. A.M. conducted the AFM topographies and the SEM images. L.Z. and D.N. addressed the local velocity profiles and proposed the FW assumption. All authors contributed to the writing of the article.

\section{Additional information}

Supplementary Information accompanies this paper at https://doi.org/10.1038/s41467018-03642-w.

Competing interests: The authors declare no competing interests.

Reprints and permission information is available online at http://npg.nature.com/ reprintsandpermissions/
Publisher's note: Springer Nature remains neutral with regard to jurisdictional claims in published maps and institutional affiliations.

(c) (i) Open Access This article is licensed under a Creative Commons Attribution 4.0 International License, which permits use, sharing, adaptation, distribution and reproduction in any medium or format, as long as you give appropriate credit to the original author(s) and the source, provide a link to the Creative Commons license, and indicate if changes were made. The images or other third party material in this article are included in the article's Creative Commons license, unless indicated otherwise in a credit line to the material. If material is not included in the article's Creative Commons license and your intended use is not permitted by statutory regulation or exceeds the permitted use, you will need to obtain permission directly from the copyright holder. To view a copy of this license, visit http://creativecommons.org/ licenses/by/4.0/.

(C) The Author(s) 2018 